\title{
GRAINS IN DIFFUSE CLOUDS: CARBON-COATED SILICATE CORES
}

\author{
DAVID A. WILliams \\ Department of Mathematics \\ UMIST \\ Manchester M60 1QD \\ United Kingdom
}

\begin{abstract}
A new model of interstellar grains is proposed in which grains in diffuse clouds consist of small (radius $\lesssim 10 \mathrm{~nm})$ and large asymmetric $(0.05 \mu \mathrm{m}-$ $0.25 \mu \mathrm{m}$ ) silicate cores thinly coated with mantles of amorphous carbon (thickness $\lesssim 5 \mathrm{~nm}$ ). This model can account successfully for many of the observed properties of interstellar dust and gives a simple interpretation of the interstellar extinction curve. The extinction bump at $220 \mathrm{~nm}$ is attributed to absorption by finely divided silicates, as indicated by laboratory data. The large silicates provide a "grey" background extinction through the visual and UV, but the bulk of the extinction in this region is attributed, on the basis of laboratory data, to the amorphous carbon coatings. The average interstellar carbon depletion required in this model is about $50 \%$.
\end{abstract}

Wide variations in the observed interstellar extinction along different lines of sight are well known. These variations have a natural explanation in this model in terms of two parameters: the thickness of the carbon coatings, and the proportion of graphitic to diamond-like structure within the amorphous carbon. The underlying silicate cores are generally unchanged in these variations. The average interstellar extinction curve requires roughly equal proportions of graphitic and diamond-like forms of amorphous carbon. A higher graphitic fraction produces more visual extinction and a higher diamond-like fraction produces more far UV extinction. Varying both the proportions, and the total carbon content encompasses a wide range of extinction curve shapes.

Amorphous carbon deposited at low temperature is generally diamond-like. Temperature excursions in the material reduce the hydrogen content and enhance the graphitic nature of the material. In the interstellar medium, this process has a time scale $\gtrsim 10^{6} \mathrm{yr}$. Carbon coatings are therefore expected to be deposited in the interstellar medium in diamond-like form and to be slowly converted to graphitelike. The growth of carbon mantles will be reversed by intermittent shocks Thus, interstellar space should contain grains with a range of thicknesses of carbon coatings, and compositions between graphitic and diamond-like.

The chemical and physical properties of amorphous carbon have been the subject of intensive laboratory study. These properties enable an understanding of a variety 
of observations of dust (especially in the IR) and lead to a number of predictions which are described in this paper.

\section{INTRODUCTION}

For many amorphous materials the coefficient, $\propto$, for absorption of radiation of energy $E$ is found to obey the relation:

$$
\propto E=B\left(E-E_{g}\right)^{2}
$$

where $E_{g}$ is the bandgap energy, and $B$ is a constant of the material (Mott and Davis, 1979). Duley (1987) has shown that, for the interstellar situation in which $\propto$ may be replaced by the extinction $A_{\lambda}$ and $E$ by $1 / \lambda$, that $\left(A_{\lambda} / \lambda\right)^{\frac{1}{2}}$ plotted against $1 / \lambda$ gives two approximately linear portions of similar slope, intercepting the $1 / \lambda$ (or energy) axis at about $0.3 \mathrm{eV}$ and $2.5 \mathrm{eV}$. The implications of this finding are:

1. that interstellar grains are amorphous;

2. that the materials providing the two separate linear portions are the same (since the slopes are the same);

3. that this material can be identified by the inferred bandgaps to be amorphous carbon. Robertson and O'Reilly (1987) have shown that amorphous carbon has two forms: one is "graphitic" (in which no long range order exists) with bandgap about $0.3 e \mathrm{e}$, and one is "diamond-like" (again, with no long range order) with bandgap about $2.5 \mathrm{eV}$. The further implication is, therefore:

4. that much of the interstellar extinction curve is due to amorphous carbon. Duley (1987), using his (1984) measured optical constants, has shown that the observed extinction is indeed consistent with this interpretation.

Amorphous carbon in the interstellar medium will become partially hydrogenated; such material is sometimes referred to as HAC. However, few amorphous carbons that have been studied in the laboratory show absorption near 220nm. By contrast absorption in finely divided silicates (specifically $\mathrm{Mg}_{2} \mathrm{SiO}_{4}$ ) has a profile that is a very close match to the well-known astronomical feature at that wavelength (Steel and Duley, 1987). The feature is attributed to $O H^{-}$ions at defects in the silicate structure. It is an assumption of the model that small silicate cores are responsible for the interstellar extinction peak at $220 \mathrm{~nm}$. Large, iron-rich, asymmetric silicate cores are assumed to be responsible for visual polarization. Table 1 summarizes the assignments of the model to the various visual and UV observed features. 
TABLE 1

Assignments of the MOdel to Extinction Features

\begin{tabular}{lll}
\hline \hline amorphous carbon mantles & $:$ & visual and UV extinction \\
small silicates $\left(\mathrm{Mg}_{2} \mathrm{SiO}_{4}\right)$ & $:$ & $220 \mathrm{~nm}$ extinction bump \\
$\begin{array}{l}\text { large, asymmetric, iron-rich, } \\
\text { silicate cores }\end{array}$ & $:$ & $\begin{array}{l}\text { grey extinction, visual } \\
\text { polarization }\end{array}$ \\
\hline
\end{tabular}

\section{VISUAL AND UV AVERAGE EXTINCTION AND POLARIZATION PROP- ERTIES}

We adopt for the silicate cores the measured optical constants of basaltic glass (Pollack et al., 1973; Lamy, 1978): this is a representative amorphous silicate which has fairly broad and smooth features in the IR near 10 and $20 \mu \mathrm{m}$. The refractive indices used for amorphous carbon are those measured by Duley (1984) which apply to material with approximately equal proportions of "graphitic" and "diamond-like" allomorphs. Calculations of extinction using Mie Theory supplemented by a small particle approximation (Duley, Jones and Williams, 1988) were made, and fits to the average interstellar extinction curve and polarization variation were obtained by varying grain sizes and mantle thickness. The model fits obtained are given in Table 2.

TAble 2: Parameters of the MODEl, Determined From FITTING INTERSTELLAR EXTINCTION AND POLARIZATION

\begin{tabular}{lcccc}
\hline \multicolumn{1}{c}{ Grain Component } & Core radius & Mantle thickness* & \multicolumn{2}{c}{ Mass ratio } \\
& & & Silicate & HAC \\
\hline $\begin{array}{l}\text { Small, HAC coated } \\
M g \text {-rich silicate cores }\end{array}$ & $<10 \mathrm{~nm}$ & $3 \mathrm{~nm}$ & 0.35 & 0.5 \\
$\begin{array}{l}\text { Large, HAC coated } \\
F e-\text {-rich aspherical } \\
\text { silicate cores }\end{array}$ & $0.05 \mu \mathrm{m}-0.25 \mu \mathrm{m}$ & $3 \mathrm{~nm}$ & 0.65 & 0.5 \\
\hline
\end{tabular}

* fraction of total available carbon in mantles, $50 \%$

Assumptions: HAC density $=1.6 \mathrm{~g} \mathrm{~cm}^{-3}$, Silicate density $=2.5 \mathrm{~g} \mathrm{~cm}^{-3}$

With these assumptions, a satisfactory fit to the interstellar extinction curve can be obtained (see Fig. 2 in Jones, Duley and Williams, 1987). The extinction is dominated by the HAC contribution at most wavelengths. The fit to the polarization observations of Wilking, Lebofsky and Rieke (1982) is illustrated in Duley et al. (1988). 


\section{EXTINCTION VARIATIONS}

Extinction curves along different lines of sight in the Galaxy show considerable variations (cf. Witt, Bohlin and Stecher, 1984), and various correlations or noncorrelations between different parts of the curve (visual, bump, far UV) have been noted, (see Jones et al., 1987 for a discussion). In the present model, it is assumed that while the silicate cores are stable refractory particles destroyed only by rare fast shocks, the amorphous carbon mantles are destroyed more readily by moderate velocity $\left(10-20 \mathrm{~km} \mathrm{~s}^{-1}\right)$ shocks or by chemical action. Further, as we shall discuss below, the nature of the amorphous carbon is not immutable, but will respond to changes in the interstellar environment. Thus, the graphitic: diamond-like ratio may change. Jones et al. (1987) have shown that the peculiar diffuse cloud extinction curves of Witt et al. (1984) can, in principle, be accounted for by variations in the thickness of the amorphous carbon mantle and its graphitic: diamond-like balance. This conclusion remains, however, somewhat tentative until further studies of the optical constants of amorphous carbon are made in the far UV.

However, with this model, the various correlations and anti-correlations observed have a natural explanation. In particular, Carnochan's (1986) deduction of an anti-correlation between the extinction at $274 \mathrm{~nm}$ and the slope of the extinction continuum from $140 \mathrm{~nm}$ to $274 \mathrm{~nm}$ is a natural consequence of this model (see Jones et al., 1987). Steeper slopes imply higher than normal carbon depletion and thicker mantles, and thus the observation that stars with steeper extinction slopes tend to have weaker $220 \mathrm{~nm}$ features (Carnochan, 1986) is also a natural consequence of this model (Jones et al., 1987).

\section{CONSEQUENCES OF THE MODEL IN THE VISUAL AND UV}

Absorption due to amorphous carbon dominates the far UV extinction (Duley, 1987). Thus, we may use the far UV extinction measurements to predict carbon depletion in the interstellar medium, after making allowance for the silicate contributions. Duley et al. (1988) have shown that at $\lambda^{-1}=8 \mu \mathrm{m}^{-1}$ (i. e., $\lambda=125 \mathrm{~nm}$ ) the fraction, $\delta_{C}$, of carbon in solid form can be written:

$$
\delta_{C} \simeq 0.07\left[\frac{E(125-V)}{E(B-V)}+1.08\right]
$$

where the colours can be measured (cf. Witt et al., 1984). Then $\delta_{C}$ for the galactic average extinction curve is predicted by equation (2) to be 0.5 , and the range for the peculiar stars of Witt et al. is $0.2 \rightarrow 0.8$.

Similarly, the strength of the bump can be calculated on the basis of this model in an unambiguous way by allowing for contributions to extinction at $217 \mathrm{~nm}$ from amorphous carbon and large silicate grains. Duley et al. (1988) have shown that one can write:

$$
E(B U M P)=0.6 \frac{E(217-V)}{E(B-V)}-5.24 \delta_{C}+0.66
$$

However, until reliable determinations of carbon depletion are available it will not be possible to test the prediction of equation (3). Equations (2) and (3) can, 
in principle, be combined to give a relation between the bump strength and other extinction related parameters. This test has not yet been performed.

The amorphous carbon contains within its structure small ordered regions (Robertson and O'Reilly, 1987) which possess some of the properties of Platt particles (Platt, 1956). In particular, these "islands" have a finite probability of re-radiating following absorption at the same wavelength. For atoms, this probability is generally unity, but the probability is smaller for larger systems. Thus, there is a contribution to the albedo of grains from these resonantly scattering systems, as was inferred by Witt, Walker, Bohlin and Stecher (1982). The inferred value of the average interstellar albedo requires that the probability for resonant scattering by the islands of amorphous carbon is about 0.4 in the visual and 0.6 in the UV.

\section{PROPERTIES OF HAC: CONSEQUENCES IN THE RED AND INFRARED}

HAC is an efficient luminescent material when excited by radiation at energies exceeding 2.5eV (Watanabe, Hasegawa and Kurata, 1982). This luminescent emission is attributed to recombination of electron-hole pairs across a bandgap in the material and is therefore controlled by the bandgap energy in the material. Diamond-like amorphous carbon has a bandgap at about $2.5 \mathrm{eV}$, as we have seen already (Robertson and O'Reilly, 1987), and thus luminescence at wavelengths around $600 \mathrm{~nm}$ is to be expected from such material in the interstellar medium. The broad band emission observed in the Red Rectangle (HD 44179) (Cohen et al., 1975; Schmidt, Cohen and Margon, 1980; Warren-Smith, Scarrott and Murdin, 1981) has been shown by Duley (1985) to be well matched by the luminescence spectrum of HAC at a temperature of $81 \mathrm{~K}$ obtained by Watanabe et al. (1982). Similar red emission has been observed in some other astronomical sources, and a diffuse emission (around 550nm) may have been detected from the general interstellar medium (van Breda and Whittet, 1981).

The bandgap energy depends on the nature of the amorphous carbon. Freshly deposited amorphous carbon has a high hydrogen content and a large bandgap (Watanabe et al., 1982; Dischler et al., 1983) Such carbon films are subject to photodarkening on exposure to UV light, and this conversion is accompanied by a release of $H$ atoms and a reduction in the bandgap energy. Duley and Williams (1988a) infer that amorphous carbon is deposited in the interstellar medium largely in a diamond-like form (bandgap 2.5eV) and that under irradiation by UV light (over a period on the order of a million years) this material in converted to graphitic form (though still amorphous). Material with a bandgap about $0.3 \mathrm{eV}$ will be expected to luminesce in the infrared and thus contribute to the near infrared continuum observed in astronomical objects (Duley and Williams, 1988a).

The amorphous nature of the solid carbon affects in a significant way the optical properties of the material. Robertson and O'Reilly (1987) have shown that amorphous carbon can be considered as a collection of molecular clusters loosely aggregated to form an extended solid. The clusters are relatively poorly connected, mechanically, electrically, and thermally. Duley and Williams (1988b) have proposed that such material is capable of thermal spiking, i.e. radiation absorbed by a single cluster is shared with neighbouring clusters (but not necessarily with the entire solid) before radiation occurs. The energy localization leads to elevated local temperatures, while the bulk remains at low temperature, so that radiation char- 
acteristic of high temperature is emitted from the hot spot. This phenomenon is used to describe the effects of radiation damage in amorphous solids; Phillips (1982) has shown that the thermal spikes can exceed $800 \mathrm{~K}$ and the excitation can persist for up to $0.01 \mathrm{~s}$. Duley and Williams $(1988 b)$ conclude that the amorphous carbon grains will therefore be important contributors to the infrared emission and that large numbers of very small grains are not required to account for this emission (cf. Draine and Anderson, 1985). The implications with regard to the unidentified infrared bands and continuum infrared emissions have been discussed by Duley, 1989.

\section{CONCLUSIONS, PREDICTIONS AND IMPLICATIONS}

The model presented here accounts in a natural way not only for the average interstellar extinction and polarization curves but also for the variety of extinction curves observed, for red and infrared continuum emission, and discrete IR features.

The model has great predictive power. It makes an immediate prediction of carbon depletion along individual lines of sight. Such predictions will be tested in the near future by the Space Telescope. The model proposes a relation (equation 3 ) between the strength of the extinction bump at $220 \mathrm{~nm}$ and carbon depletion which is similarly open for testing. The model requires that regions of exceptionally strong far UV extinction will also be sources of extended red emission. Since the bandgap energy in diamond-like carbon increases with $H$ content, the model enables a prediction of the dust $H$ content in red emission regions, and allows a calibration of this $H$ content by the peak wavelengths of the emission. The $9.7 \mu \mathrm{m}$ and $18 \mu \mathrm{m}$ silicate features are predicted to have components from both iron and magnesium silicates. However, the polarization at these wavelengths will have only one component, due to the iron-rich silicate. The $220 \mathrm{~nm}$ extinction bump, on the other hand, arises from the small magnesium silicate cores, and should therefore be unpolarized. The model accounts for the general interstellar IR band and continuum emission without invoking large numbers of very small grains. In particular, high fractions of available carbon in the form of free-flying polycyclic aromatic hydrocarbons (PAHs) are not required. High temperature emission can occur from bulk grain material; the counterpart of this phenomenon is that some of the energy must leak out as $3.4 \mu \mathrm{m}$ emission: it is predicted that there is a general background emission at this wavelength in the Galaxy.

The implication of the model is exciting. Grains are not inert, passive objects in the interstellar gas; they respond to and change with their environment. Freshly deposited carbon is diamond-like and is annealed to the more stable graphitic form in a few million years (typically). This is also the typical timescale for carbon deposition on the silicate cores. Some processes, possibly intermittent low velocity shocks, must occur to limit the carbon mantle growth and return the carbon to the gas. Thus, the model implies a continual recycling of carbon into and out of solid form on a time scale of a few million years. Such a process will have profound consequences for interstellar gas phase processes.

ACKNOWLEDGEMENTS. This work has been supported by grants from SERC and NSERCC. I am grateful to W. W. Duley and A. P. Jones for many stimulating 
and informative discussions.

\section{REFERENCES}

Carncochan, D. J. 1986, M. N. R. A. S., 219, 903.

Cohen, M. et al. 1975, Ap. J., 196, 179.

Dischler, B., Bubenzer, A. \& Koidl, P. 1983, Appl. Phys. Lett., 42, 636.

Draine, B. T. \& Anderson, N. 1985, Ap. J., 292, 494.

Duley, W. W. 1984, Ap. J.,287, 694 .

. $1987, M . N . R$. A. S., $229,203$.

Duley, W. W., Jones, A. P. \& Williams, D. A. 1988, M. N. R. A. S., in press.

Duley, W. W., \& Williams, D. A. 1988a, M. N. R. A. S.,230, 1 P. $1988 b, M . N . R$. A. S.,231, 969P.

Duley, W. W. 1989, in IAU Symposium 195, Interstellar Dust, eds. L. J. Allamandola and A. G. G. M. Tielens, (Dordrecht: Kluwer), p. 141.

Jones, A. P., Duley, W. W. \& Williams, D. A. 1987, M. N. R. A. S., 229, 213.

Lamy, P. L. 1978, Icarus, \$4, 68.

Mott, N. F. \& Davis, E. A. 1979, in Electronic Processes in Non-Crystalline Materials, (Oxford: Clarendon Press).

Phillips, J. C. 1982, Phys. Rev. B, 25, 1397.

Platt, J. R. 1956, Ap. J., 123, 486.

Pollack, J. B., Toon, O. B. \& Khare, B. N. 1973, Icarus, 19, 372.

Robertson, J.' \& O'Reilly, E. P. 1987, Phys. Rev. B, 35, 2946.

Schmidt, G. D., Cohen, M. \& Margon, B. 1980, Ap. J., 239, 133.

van Breda, I. G. \& Whittet, D. C. B. $1981, M$. N. R. A. S., 195, 79

Warren-Smith, R. F., Scarrott, S. M. \& Murdin, P. 1981, Nature, 292, 317.

Watanabe, I., Hasagawa, S. M. \& Kurata, Y. 1982, Japan J. Appl. Phys., $21,856$.

Wilking, B. A., Lebofsky, M. J. \& Rieke, G. H. 1982, Ap. J., 87, 695.

Witt, A. N., Walker, G. A. H., Bohlin, R. C. \& Stecher, T. P. 1982, Ap. J., $261,492$.

Witt, A. N., Bohlin, R. C. \& Stecher, T. P. 1982, Ap. J., 279, 698. 